\title{
Von Gentrifizierung betroffen
}

Ein exemplarischer Beitrag zur Diskussion konzeptioneller und methodischmethodologischer Fragen qualitativer Verdrängungsforschung

\author{
Miriam Meuth \\ Christian Reutlinger
}

\begin{abstract}
Verdrängung als Teil von Gentrifizierungsprozessen stellt einen wichtigen und noch stärker zu erforschenden Aspekt einer kritischen Stadt- und Wohnforschung dar. Zur Erforschung der subjektiven Deutungen von Verdrängung bietet sich ein qualitativer Zugang an. Neben konzeptionellen und methodisch-methodologischen Fragen und Diskussionen gilt es, dieses Phänomen auch differenziert empirisch zu beleuchten, nicht zuletzt unter Berücksichtigung der Perspektiven, des Erlebens und auch des Umgangs der von Verdrängung bedrohten oder betroffenen Menschen. Im Beitrag verfolgen wir vor diesem Hintergrund die Frage, welche konzeptionellen und methodisch-methodologischen Herausforderungen und welche möglichen Lösungswege sich für einen qualitativen Zugang zu Verdrängung ergeben. Mit der Diskussion dieser Fragen möchten wir ausgehend von einem jüngst abgeschlossenen Forschungsprojekt einen exemplarischen Beitrag zur Auseinandersetzung mit Methoden und Erkenntniswegen qualitativer Verdrängungsforschung im Kontext von Gentrifizierung leisten.
\end{abstract}

Ersteinreichung: 22. November 2019; Veröffentlichung online: 23. April 2021

An English abstract can be found at the end of the document.

\section{Einleitung}

Medial werden sozial- und wohnungspolitische Missstände und die negativen Folgen für Mieter_innen, die unter prekären Wohnbedingungen leben oder ihre Wohnung verlieren, gegenwärtig auf vielen Kanälen angeprangert.[1] Es sind soziale Bewegungen, aber auch der wissenschaftliche Diskurs, die über die hinter den Gentrifizierungsprozessen liegenden strukturellen Mechanismen und der damit einhergehenden Verdrängung der bisherigen Mieter_innen aufklären.

Historisch und gesellschaftlich sind die skizzierten Entwicklungen gerahmt durch die Tatsache, dass Städte seit zwei Jahrzehnten wieder mehr Zuzug erleben; ein Trend, der in der Stadtforschung in Deutschland, aber auch in der Schweiz unter dem Schlagwort der Reurbanisierung (Brake/ Herfert 2012) oder dem „Trend der Metropolitanisierung“(Helbrecht 2016:9) diskutiert wird. Dies hat jedoch Folgen für den Mietmarkt: „Mit der Zahl der Menschen nimmt auch die Nachfrage nach Wohnraum zu, während das Wohnungsangebot insbesondere in den Innenstädten der Metropolen bei Weitem nicht im selben Maße mitwächst." (Nuissl/Beran 2019: 8) Angetrieben wird dieser Prozess aktuell auch durch eine Nullzinspolitik, die wiederum 
zu verstärkten Investitionen in Wohnraum führt, in deren Zentrum nicht mehr der Nutzen von Wohnungen, sondern ihr Renditepotenzial steht; diesbezüglich ist von der „Finanzialisierung von Immobilien“ (Heeg 2013: 75) die Rede. Das bedeutet, dass in solchen Wohnvierteln beziehungsweise -siedlungen investiert wird, in denen der Gewinn am größten ist. So stellt Verdrängung keinen Nebeneffekt, sondern die Bedingung von Aufwertung und Erneuerung dar (Holm 2011: 12; Marcuse 1985). Die durch Verdrängung ausgelösten Veränderungen in der Zusammensetzung der Bewohner_innenschaft hinsichtlich sozioökonomischer Merkmale sind mitunter Effekte der baulichen Aufwertung, da damit in der Regel bezahlbare (Miet-)Wohnungen reduziert werden, die aus investitionsstrategischer Sicht nicht von Interesse sind (Holm 2011: 12). Nicht zuletzt dadurch werden Mieter_innen aus ihren Wohnungen, aber auch aus der Nachbarschaft in unterschiedlichen Ausprägungen und Formen verdrängt. Im Diskurs um Gentrifizierung wird entsprechend gefordert, die Sicht auf die und aus der Perspektive der Verdrängten zu erforschen (Holm 2013: 59).

Insofern stellt die in den letzten Jahren vermehrte empirische Untersuchung von Verdrängung im Kontext von Gentrifizierung keine zufällige Entwicklung dar, sondern kann als wissenschaftliche Reaktion auf ein immer präsenter werdendes gesellschaftliches Phänomen gedeutet werden.

In vielen städtisch geprägten Siedlungsgebieten spitzt sich die Lage auf dem Wohnungsmarkt massiv zu - auch im dicht besiedelten Schweizer Mittelland, auf das sich die empirische Studie, die diesem Artikel zugrunde liegt, bezieht. Die Totalsanierung von Wohnblöcken beziehungsweise deren Abriss und der Bau neuer Häuser verbunden mit der Aufwertung ganzer Siedlungen steht hierzulande im Zeichen von politisch forcierter baulicher und sozialer Verdichtungsbestrebungen. Über die Beobachtung, dass es im Zuge dieser Aufwertungs- und Neubauprozesse zur Kündigung sämtlicher Mietverhältnisse in den Siedlungen kommt - sogenannten Leerkündigungen -, gelangen wir auf indirektem Weg zu einem zentralen Thema in der Wohn- und Stadtforschung, der Verdrängung von Mieter_innen: Verdrängung verstehen wir vor dem Hintergrund unserer empirischen Fallstudie als durch Wohnungskündigung erzwungenen Auszug. Die Haushaltsmitglieder können die Gründe für den Auszug weder kontrollieren noch verhindern, sie werden fremdbestimmt damit konfrontiert und ihnen bleibt nur, irgendwie damit umzugehen (Grier/Grier 1980: 256). Damit liegt unser Erkenntnisinteresse auf direkter Verdrängung („direct displacement“; Marcuse 1985: 205). Auch wenn die Verdrängung aus einer Wohnung oder Nachbarschaft vielerlei Ursachen haben kann, zum Beispiel eine Naturkatastrophe oder den Bau einer Autobahn, fokussieren wir in diesem Beitrag auf Verdrängung im Zusammenhang mit Gentrifizierung (gentrification-induced displacement) sowie auf den forscherischen Zugang zu diesem Phänomen.[2] Gentrifizierung verstehen wir im Weiteren als „,jede[n] stadtteilbezogene[n] Aufwertungsprozess, bei dem immobilienwirtschaftliche Strategien der Inwertsetzung und/oder politische Strategien der Aufwertung den Austausch der Bevölkerung“(Holm 2018: 152) voraussetzen.

Auch wenn die folgenden Überlegungen von einer spezifischen Form direkter Verdrängung aus getätigt werden, können sie für die Erforschung anderer Formen von Verdrängung inspirierend sein, sofern sie ausgehend 
von den jeweiligen spezifischen Fragestellungen und Fallbeispielen ausdifferenziert und angepasst werden.

Nicht nur weil Verdrängung aktuell auf maßgebliche Weise städtische soziale Ungleichheit veranschaulicht, sondern auch, weil sich für die Verdrängungsforschung analytisch-konzeptionelle Fragen stellen, möchten wir mit diesem Artikel einen exemplarischen Beitrag zu konzeptionellen und methodisch-methodologischen Fragen und Herausforderungen sowie möglichen Lösungswegen für einen qualitativen Zugang zu Verdrängung leisten.

Dabei gehen wir wie folgt vor: Zunächst skizzieren wir, wie Verdrängung in der Literatur diskutiert wird und welche Fragen sowie Herausforderungen sich konzeptionell, aber auch für die Erforschung des Phänomens ergeben (Kapitel 2). Dann stellen wir das Forschungsprojekt WOHNSOG sowie dessen Kontextualisierung in der Deutschschweiz vor (Kapitel 3). Damit ist der Projektzusammenhang expliziert, vor dessen Hintergrund wir in Kapitel 4 konzeptionelle, methodische und methodologische Schlussfolgerungen unter Berücksichtigung der in Kapitel 2 dargelegten Verunsicherungen der Verdrängungsforschung darlegen. Im Fazit (Kapitel 5) fassen wir unser Vorgehen kurz zusammen und argumentieren dafür, die unterschiedlichen Forschungsdesigns nicht gegeneinander auszuspielen, sondern sie gegenstandsangemessen zu begründen. Schließlich legen wir dar, warum unserer Ansicht nach eine differenzierte und kritisch geführte Verdrängungsforschung gerade unter den aktuellen wohnpolitischen, aber auch den wissenschaftlich diskursiven Auseinandersetzungen notwendig ist.

Mit dem vorliegenden Artikel möchten wir Denkanstöße und Lösungswege für zukünftige Projekte qualitativer Forschung bieten, die sich mit dem aktuell hoch relevanten Thema der Verdrängung von Mieter_innen beschäftigen und besonderes Augenmerk auf die konzeptionellen und methodischen-methodologischen Herausforderungen einer qualitativen Verdrängungsforschung legen.

\section{Verdrängung erforschen}

Ein Blick in die internationale Gentrifizierungsforschung zeigt, dass Aspekte der Verdrängung gegenüber den Ursachen, Prozessen, Akteur_innen und Verläufen von Gentrifizierung sowie dem Agieren der Immobilienwirtschaft verhältnismäßig wenig erforscht werden (Helbrecht 2016: 9 f.). Zwar ist Verdrängung von Anbeginn der Gentrifizierungsforschung Thema, sie gerät aber phasenweise mal mehr und mal weniger ins Zentrum der Forschung (Pull et al. 2021: 5 ff.). Neben Gründen, die im wissenschaftlichen Diskurs liegen, spielt hier sicher auch die gesellschaftspolitische zeitweise (De-)Thematisierung von Verdrängung im städtischen Leben eine wichtige Rolle. Es lässt sich festhalten, dass Verdrängung relativ betrachtet bisher vernachlässigt wird, vor allem was die Berücksichtigung der Perspektiven der Verdrängten betrifft. Dies scheint sich jedoch mit Blick auf aktuelle Studien zu verändern.[3]

Diese Feststellung wird in der Literatur zu Gentrifizierung und Verdrängung unter anderem auf konzeptionelle, aber auch auf methodische Herausforderungen und methodologische Fragen zurückgeführt (Easton et al. 2019; Helbrecht 2016: 13; Holm 2013: 59; Lees/Slater/Wyly 2010). Hervorgehoben wird, dass bereits die Definitionen und Operationalisierungen von 
Verdrängung und Gentrifizierung den Zugang zum Gegenstand bedingen und politische Implikationen haben (Helbrecht 2016). Ebenso wird die empirische Operationalisierung des Verdrängungskonzepts kritisiert, Letzteres werde sehr unterschiedlich verwendet und oftmals unpräzise operationalisiert (Beran/Nuissl 2019b: 19). Es wird konstatiert, dass es herausfordernd sei, Verdrängung „exakt zu definieren und - vor allem - zu messen“(Nuissl/ Beran 2019: 9; siehe auch Atkinson 2000; Easton et al. 2019).

Angemerkt wird zudem, dass der Zugang zu Verdrängten äußerst schwierig sei und dieser eher gelinge, wenn sich Verdrängung bereits manifestiert hat. Spezifische Formen von Verdrängung seien - was den forschungspraktischen Zugang angeht - also besser zu erforschen als andere (Helbrecht 2016: 13; Holm 2013: 60). Hier sei des Weiteren darauf zu achten, wen man in der Forschung überhaupt erreicht und wen nicht. So liegt die These eines Bias in den Daten nahe, denn sprachbasierte Forschungsdesigns, sei es in Fragebögen oder in qualitativen Interviews, setzen spezifische Ressourcen zur Teilnahme voraus.

Außerdem stellen sich ethische Fragen danach, wie der vulnerablen Situation der Betroffenen im Forschungsprozess Rechnung getragen werden kann. Quantitative wie qualitative Längsschnittstudien (Holm 2013: 59; Easton et al. 2019: 14) oder auch wohnbiografische Zugänge (Betancourt 2016) in diesem Bereich wären hilfreich, um Verdrängung nicht nur punktuell und retrospektiv, sondern über einen längeren Zeitraum untersuchen zu können. Die Herausforderung, mikro- und makroanalytische Zugänge zu verbinden, wird diskutiert (Sakizlioğlu 2014). Schließlich erweist sich eine reflexive Haltung mit einem Bewusstsein für die eigene Positionalität im Forschungsprozess als für die Verdrängungsforschung bedeutsam. Und mehr noch, gerade diese Bedingungen der eigenen Positionen der Forscher_innen und die damit einhergehenden blinden Flecken werden in der Literatur als Erklärung für die empirische Vernachlässigung in der Gentrifizierungsforschung herangezogen. So besteht die These der bildungsbürgerlichen Ferne von Forscher_innen zu den „Verlierer_innen der Gentrification“ (Holm 2013: 59; Allen 2008). Andernorts ist in eine ähnliche Richtung argumentierend von der Stadtforschung als einem „einäugigen Zyklopen“ und von einer ,immensen intellektuellen Einseitigkeit“ die Rede (Helbrecht 2016: 11). Vor diesem Hintergrund plädiert Allen (2008: 180) dafür, dass Konflikte zwischen den unterschiedlichen Perspektiven derjenigen, die Gentrifizierung erforschen, und derjenigen, die deren Konsequenzen erleben, sichtbar zu machen seien. Er spricht von einer fehlenden „referential reflexivity“, mit der die Unfähigkeit einhergehe, die ontologische Rolle der eigenen Positionalität zu reflektieren.

Der Blick in die internationale Gentrifizierungsforschung zeigt schließlich, dass mittels qualitativer Forschungsdesigns häufig gerade das Erkenntnisinteresse an der Perspektive der von Verdrängung betroffenen oder von ihr bedrohten Menschen verfolgt wird. Im Diskurs um Gentrifizierung wird entsprechend gefordert, auch die Perspektive von Verdrängten zu erforschen (Holm 2013: 59). Das heißt, dass sich ein solcher methodisch-methodologischer Zugang unter Berücksichtigung des jeweiligen Erkenntnisinteresses oftmals als gegenstandsangemessen zu erweisen scheint. Mit solchen qualitativen Forschungsdesigns kann Verdrängung aus einer „Froschperspektive“ (Eckardt 2018: 22) beleuchtet werden - also unterhalb einer Normalperspektive - eben aus der Perspektive der Betroffenen 
selbst. Je nach methodischem und methodologischem Design, das durch das Erkenntnisinteresse der Studien bedingt ist, geraten somit unter anderem subjektive Deutungen, Sinnkonstruktionen, Erfahrungen oder auch Praktiken ins Zentrum der Aufmerksamkeit. Derart designte Studien leisten eine vertiefende Innensicht auf Verdrängung und die Perspektiven der von Verdrängung bedrohten oder betroffenen Personen. Im vorliegenden Artikel fokussieren wir deshalb und aufgrund der eigenen Forschungsexpertise auf Fragen und Herausforderungen eines qualitativen Forschungszugangs zu Verdrängung.

Nicht unerwähnt lassen wollen wir die Grenzen qualitativer Verdrängungsstudien, die offenbar werden, wenn es zum Beispiel um das Ausmaß oder die Verbreitung von Verdrängung geht. Für eine solche „VogelflugPerspektive, die mit statistischen Analysen große städtische Räume betrachtet“ (Eckardt 2018: 22), sind quantitative Zugänge adäquat (Easton et al. 2019; Friedrichs 1996; Wüstenrot Stiftung 2019).

Bis hierher sollte deutlich geworden sein, dass Verdrängung - gerade weil es sich dabei um ein alltagspraktisch relevantes Phänomen handelt, von dem immer mehr Menschen betroffen sind - dezidiert und systematisch ins Zentrum der (Gentrifizierungs-)Forschung zu rücken ist. Indem wir dargelegt haben, welche Gründe in der Literatur für die relative forscherische Vernachlässigung des Gegenstandes herangezogen werden, haben wir zugleich wichtige konzeptionelle wie methodisch-methodologische Herausforderungen für die Erforschung von Verdrängung ins Bewusstsein gerufen. Dies, ohne Details und spezielle Konstellationen von Verdrängung, einzelne Fragestellungen oder auch Forschungsdesigns näher zu betrachten. Wendet man sich den skizzierten Herausforderungen im Detail zu, so findet sich dafür unserer Ansicht nach kein einfaches methodisch-methodologisches ,Rezeptwissen“ oder eine pauschale Lösung. Vielmehr gilt es, diese allgemein benannten Schwierigkeiten der Verdrängungsforschung spezifisch und ausgehend von den jeweiligen konkreten Fragestellungen, dem Erkenntnisinteresse sowie der einzelnen Kontexte empirischer Beispiele zu betrachten und zu diskutieren.

Damit wird deutlich, dass neben den bereits dargelegten allgemeinen Fragen und Herausforderungen die für diesen Artikel leitende Fragestellung nach den konzeptionellen und methodisch-methodologischen Herausforderungen sowie nach möglichen Lösungswegen für einen qualitativen Zugang zu Verdrängung nicht ohne das entsprechende Erkenntnisinteresse sowie das Forschungsdesign diskutiert werden kann. Somit können die erkenntnistheoretischen und methodisch-methodologischen Überlegungen immer nur exemplarisch und unter Rückgriff auf den jeweiligen Forschungszusammenhang diskutiert werden.

Die weiteren Ausführungen stehen insofern in Verbindung mit den bereits dargelegten Diskursen, haben jedoch ihren Ausgangspunkt in Problemstellungen der jüngst abgeschlossenen und vom Schweizerischen Nationalfonds geförderten qualitativen Studie, die im Folgenden genauer vorgestellt werden soll.

\section{Zum Forschungsprojekt WOHNSOG und zur Kontextuali- sierung in der Schweiz}

Im Zentrum des Forschungsprojektes „Bewältigung von drohendem Wohnungsverlust - die soziale Seite gebietsbezogener baulich-planerischer 
Strategien “(WOHNSOG) standen die Perspektiven von Menschen, die mittels Massenkündigungen aufgrund von Totalsanierungen oder Ersatzneubauten aus ihren Wohnungen verdrängt worden sind (Meuth/Reutlinger 2021; Reutlinger et al. 2019; Furrer et al. 2020).[4] Diese Form der Verdrängung stellt die betroffenen Mieter_innen vor die strukturelle Herausforderung, eine neue bezahlbare Wohnung zu finden; dies kann einen sozialen Abstieg und/oder den Verlust gefestigter sozialer Kontakte im Nahraum bedeuten, ganz abgesehen von emotionalen, psychischen oder gesundheitlichen Folgen. Damit ist Verdrängung eine Erfahrung, die nicht nur alltagspraktisch, sondern auch emotional und biografisch zu verarbeiten ist. Es liegt nahe, davon auszugehen, dass es sich hierbei um eine „Bewältigungsaufforderung“ (Knabe/Leitner 2017: 236; Meuth/Reutlinger 2020) handelt. Was dies für die betroffenen Menschen bedeutet - gewissermaßen die soziale Seite der immobilienwirtschaftlichen sowie stadt- und raumplanerischen Entwicklungen -, ist bisher weder in der Öffentlichkeit, in Kreisen der Raumplanung und Stadtentwicklung, noch im wissenschaftlichen Diskurs in größerem Maße diskutiert worden. Deshalb wurde im Forschungsprojekt WOHNSOG folgende Fragestellung verfolgt: Wie erleben Mieter_innen ihre Wohnungskündigung im Zuge baulicher Aufwertungs- und/oder Verdichtungsprozesse und wie gehen sie damit um?[5]

\section{Diskursive Rahmung und Ausgangspunkt der Studie}

,Leerkündigungen' aufgrund von Totalsanierungen und Ersatzneubauten stehen oftmals beispielhaft für bauliche Aufwertung sowie immobilienwirtschaftliche und teils (stadt-)politische Strategien, die zu deutlich sichtbarer und massiver direkter Verdrängung der bisherigen Mieter_innen führen. Teilweise nutzen institutionelle und profitorientierte Eigentümerschaften, besonders auf angespannten Wohnungsmärkten, derartig begründete, Leerkündigungen' als Legitimation, um die bestehenden Mietverhältnisse mit dem Ziel der Renditesteigerung zu kündigen (Mieterinnen- und Mieterverband Ostschweiz 2018: 7). Ein solches Vorgehen ist in der Schweiz mietrechtlich nur bedingt geschützt, sodass dadurch Vertragsverhältnisse auch ohne Fehlverhalten der Mieter_innen und ohne angekündigten Eigenbedarflegal aufgelöst werden können.

Parallel zu solchen immobilienwirtschaftlichen Aufwertungsbewegungen finden sich in der Schweiz aktuell politische Bestrebungen zu einer baulichen Verdichtung, um der Zersiedelung Einhalt zu gebieten. In diesem Zusammenhang wird verstärkt Altbestand saniert und dabei aufgestockt, erweitert oder auch abgerissen und die Parzelle neu bebaut (Hochschule Luzern 2019). Diskursiv gerahmt werden solcherlei Bau- und (Total-)Sanierungsprojekte - mit dem Ziel, eine höhere Nutzungsdichte zu erreichen unter anderem durch die nationale Raumentwicklungsstrategie (Revision des Raumplanungsgesetzes vom Dezember 2013).

Im Forschungsprojekt WOHNSOG vertreten wir die These, dass einerseits die immobilienwirtschaftlichen Aufwertungen der Regeneration (Erneuerung oder [Total-]Sanierung) und andererseits die politisch motivierten baulichen Verdichtungsbestrebungen vielerorts nicht einfach parallel stattfinden, sondern zutiefst diskursiv, aber auch baupraktisch miteinander 
verwoben sind. Es sind oftmals nicht lediglich immobilienwirtschaftliche Strategien, die Gentrifizierung hervorrufen, vielmehr sind diese mitunter eingebettet und gerahmt durch politische Entscheidungen und Prozesse, wie das Beispiel der „statemade-rental-gap“ im sozialen Wohnungsbau (Ertelt et al. 2016) oder die politischen Entscheidungen hinsichtlich einer sogenannten Verdichtung nach innen (Bundesamt für Raumentwicklung Schweiz ARE; Debrunner/Hengstermann/Gerber 2020; Debrunner/Hartmann 2020; Reutlinger 2015) zu verdeutlichen vermögen. Oder andersherum: Zunächst sind politische Strategien der Aufwertung gegeben, die dann immobilienwirtschaftliche Akteur_innen animieren, genau dort zu investieren. Man könnte also von einem politischen Vorstoß der Verdichtung nach innen sprechen, der einerseits aufgrund des Wohnraummangels in (Groß-)Städten und einer enormen Zersiedelungstendenz in der Schweiz planungs- und wohnungspolitisch sinnvoll ist, andererseits aber besonders den institutionellen Investoren legitimatorisch in die Hand spielen kann.

Gentrifizierung ist für uns vor diesem Hintergrund immer zugleich Aufwertung und Verdrängung; Helbrecht (2016: 13) spricht diesbezüglich von den „zwei Seiten der Medaille“ der Gentrifizierung. Solange die Wohnraumversorgung in überwiegendem $\mathrm{Ma} ß$ warenförmig organisiert ist (Vollmer 2019: 114), geht die Aufwertung ganzer städtischer Gebiete mit verschiedenen Formen der Verdrängung weniger kaufkräftiger Bevölkerungsgruppen einher. Dies trifft für unsere Beobachtungen in der Deutschschweiz zu, zumindest für lokal angespannte Wohnungsmärkte.[6]

\section{Methodisch-methodologisches Design}

Um subjektive Perspektiven auf Wohnungskündigungen innerhalb konkreter Prozesse zu kontextualisieren, bieten sich Fallstudien zu spezifischen Sanierungs- oder Ersatzneubauprojekten an. In diesem Sinne haben wir Fallstudien zu drei ausgewählten großen Totalsanierungs- respektive Ersatzneubauprojekten im Mietwohnungssektor im Zuge baulicher Verdichtung und/oder Aufwertung in Deutschschweizer (Groß-)Städten mit angespannten (Miet-)Wohnungsmärkten erstellt; Letzteres machen wir fest an einer geringen Leerstandsquote. Ziel ist es, vergleichbare - wenn auch unterschiedliche - Fallstrukturen und -bedingungen zu berücksichtigen, um kontrastierende Fälle (Fallebene: Siedlung/Gebiet) zu generieren. Alle betrachteten Wohnobjekte befinden sich in institutioneller Eigentümerschaft: ein in der Schweiz ansässiger, global tätiger Finanzdienstleister, eine städtische Liegenschaft und die Pensionskasse einer regionalen Bank. Die Liegenschaften der ausgewählten Fälle werden durch dafür beauftragte Verwaltungen ,bewirtschaftet'.

Das Ziel, möglichst viel planerisches, strategisches Wissen zu den Sanierungen und Ersatzneubauprojekten der Fallstudien zu erhalten, legte es nahe, den Feldzugang über die Eigentümerschaften und involvierte Projektentwickler_innen zu wählen, gewissermaßen eine vorübergehende forschungsstrategische Allianz einzugehen. Das wiederum hatte zum Nachteil, dass der Zugang zu Projekten enorm von den Eigentümerschaften abhing; ihnen kam als Gatekeeper im Forschungsprozess eine maßgebliche, steuernde Rolle zu. Der Feldzugang lieferte bereits erste Erkenntnisse zum Gegenstand: 
Insgesamt zeigte sich in den Telefongesprächen, dass sich die Eigentümerschaften in der Regel bewusst waren, dass sie heikle Kündigungspraktiken anwendeten und zudem öffentlich kritisiert wurden.

Im Gegensatz zu anderen Forschungen, die oft retrospektiv bereits vollzogene Verdrängungsprozesse untersuchen (Helbrecht 2016: 13; Holm 2013: 60), erforschten wir das Erleben und den Umgang mit der Wohnungskündigung vor oder kurz nach dem erzwungenen Umzug, also mitten im Verdrängungsprozess; deshalb war es auch wichtig, forschungsethische Fragen zu reflektieren. Zentrale Grundlage, um die leitende Forschungsfragestellung nach dem Umgang mit der Wohnungskündigung zu beantworten, stellten leitfadengestützte Interviews mit den Mieter_innen dar. Der Leitfaden diente als thematische Orientierung und wurde nicht starr abgehandelt. Vielmehr sollte sichergestellt werden, dass über alle Interviews und Interviewer_innen hinweg die gleichen Themen behandelt werden (Helfferich 2011; Meuser/Nagel 2009). In der Interviewinteraktion hingegen rückte der Leitfaden in den Hintergrund und ließ Raum für die individuellen Strukturierungen der Interviewten (Meuth 2018: 75).

Die leitfadengestützten Interviews setzten sich aus fünf Themenbereichen zusammen, die sich jeweils mittels erzählgenerierender, offener Fragen ausdifferenzierten: Wohnbiografie, Siedlung und lokaler Kontext, Netzwerke/ soziale Kontakte/Beziehungen, gekündigte Wohnsituation mit Ressourcen und Belastungen sowie Einschätzung der eigenen Rolle/Person, Perspektive und Meinung. Um die leitende Forschungsfrage umfassend beantworten zu können, waren die Leitfadeninterviews stark narrativ gehalten. So konnte neben Meinungen und Beschreibungen auch Erzählungen Raum gegeben werden (Schütze 1983, 1984). Denn im Sinne der Homologiethese, so die erzähltheoretische Annahme Schützes, kommt die Textsorte des Erzählens auch retrospektiv am nächsten an das Erlebte und macht so Empfindungen, Gefühle und Gedanken sowie Eigentheorien bezüglich der Kündigungserfahrung erfassbar. Die Interviews mit Mieter_innen wurden vollständig und systematisch anhand spezifischer Regeln (Langer 2010: 523; Przyborski/ Wohlrab-Sahr 2009: 168) transkribiert.

Ergänzend zu den Interviews, zu Zwecken der Anreicherung der Fallstudien, aber auch, um einen besseren Zugang zu Interviewpartner_innen zu erhalten, hielten wir uns jeweils mehrere Tage vor Ort auf und beobachteten. Dabei richteten wir den Fokus auf physisch-materielle Bedingungen der Siedlungen sowie auf die subjektiv wahrgenommene Atmosphäre. Die angefertigten Beobachtungsprotokolle enthalten zudem Gesprächsnotizen von Kurzgesprächen mit weiteren Mieter_innen.

Der Zugang zu den Interviewpartner_innen erfolgte auf unterschiedlichen Wegen: über Aushänge in Treppenhäusern, in einem Fall außerdem über einen Brief, der durch die Liegenschaftsverwaltung in unserem Namen verschickt wurde; in einem weiteren Fallbeispiel warfen wir selbst eine Interessensbekundung zum Interview in die Briefkästen ein; sowie über die direkte Ansprachen von Menschen, die wir während der Beobachtungen vor Ort trafen. Aufgrund des erschwerten Zugangs zu Personen, die sich in einer solch herausfordernden Situation für Forschungszwecke interviewen lassen, konnten wir für das Sample vorab keine Kriterien festlegen. Stattdessen interviewten wir alle Personen, die sich dafür bereit erklärten, und erhielten am 
Ende 20 Interviews mit Mieter_innen. Größtenteils wurden die Interviews in der bisherigen Wohnung, nur teilweise in der neuen Wohnung der Person geführt. Im Anschluss an die Interviews ließen wir jeweils einen soziodemografischen Kurzfragebogen zur Person ausfüllen und fertigten Postskripte zum Entstehungszusammenhang des Interviews, zur Kontaktaufnahme mit der Person, zur Interviewsituation, zum Ort sowie zu Reflexionen über die eigene Rolle als Interviewer_innen an.

Gerät in der qualitativen (Verdrängungs-)Forschung ein „konkretes Handlungs- oder Interaktionsphänomen“(Breuer 2010: 88) in den Blick, das prozessorientiert ist (Strauss/Corbin 1996: 23), erweist sich die Grounded Theory methodisch und methodologisch als angemessener Forschungsstil. Strauss und Corbin (1996: 83) halten diesbezüglich allgemein fest:

„Grounded Theory ist eine handlungs- und interaktionsorientierte Methode der Theorieentwicklung. Ob man Individuen, Gruppen oder Kollektive untersucht, immer gibt es Handlung und Interaktion, die auf ein Phänomen gerichtet ist, auf den Umgang mit ihm und seine Bewältigung, die Ausführung oder die Reaktion darauf, wobei das Phänomen immer in einem Kontext oder unter einem spezifischen Satz von Bedingungen auftritt.“

Mit diesem Hinweis wird deutlich, dass wir das subjektive Erleben und den Umgang mit der Wohnungskündigung der verdrängten Mieter_innen erkenntnistheoretisch fundiert nicht nur handlungstheoretisch und prozesshaft verstehen, sondern auch immer kontextualisiert betrachten. Denn die von den Interviewten dargelegten zentralen Phänomene und ihr Umgang mit ihnen finden in einem vorgegebenen Rahmen und rückgekoppelt an verschiedene kontextuelle Ebenen statt. Forschungspraktisch bedeutet dies, dass im Projekt das unterschiedliche Datenmaterial mittels diverser forschungspraktischer Techniken im Stil der Grounded Theory (Strauss/Corbin 1996) ausgewertet wurde. Neben dem mehrstufigen Kodierprozess (offenes, axiales und selektives Kodieren), bei dem es um ein theoretisches Kodieren und nicht nur um eine Klassifikation oder Sortierung und Beschreibung des untersuchten Phänomens geht (Breuer 2010: 41), orientierten wir uns am Prinzip eines abduktiven Forschungsprozesses und an der Methode des ständigen Vergleichs (zwischen verschiedenen Phänomenen, zwischen verschiedenen Kontexten, Interfallvergleich, Intrafallvergleich) (Schröer/ Schulze 2010: 282; Strübing 2010: 15).

\section{Konzeptionelle und methodisch-methodologische Schlussfolgerungen}

Nachdem wir bis hierher in der Verdrängungsforschung bestehende konzeptionelle sowie methodisch-methodologische Herausforderungen dargelegt sowie das Projekt WOHNSOG samt Ausgangslage und Forschungsdesign vorgestellt haben, diskutieren wir abschließend konzeptionelle und methodisch-methodologische Schlussfolgerungen. Von den in Kapitel 2 dargelegten Verunsicherungen in der Verdrängungsforschung greifen wir hier einige Aspekte auf: die Konzeption der Verdrängung sowie der qualitativen Operationalisierung des Umgangs mit ihr, die Kontextualisierung 
der Subjektperspektive respektive der Verbindung von mikroanalytischen Erkenntnissen mit einer Makroperspektive sowie der (Ohn-)Macht und der eigenen Positionalität im Forschungsprozess. Ausgehend von unserem Untersuchungsgegenstand und Erkenntnisinteresse sowie den erkenntnistheoretischen Prämissen, die dem Stil der Grounded Theory zugrunde liegen, zeigen wir somit Lösungswege für die Erforschung des Phänomens der direkten Verdrängung auf. Damit sind diese Schlussfolgerungen zwar übertragbar auf andere Forschungszusammenhänge, müssen jedoch gegebenenfalls angepasst, erweitert oder verändert werden.

\section{Konzeption von Verdrängung als komplexes und prozesshaftes Phänomen}

Mit Blick auf das Erkenntnisinteresse des Projekts WOHNSOG stellen wir nun konzeptionelle Überlegungen zum Verdrängungs- und Gentrifizierungsverständnis an, die verdeutlichen werden, dass diese Begriffe nicht universell zu definieren sind, sondern jeweils vor dem Hintergrund des untersuchten Falles spezifiziert werden müssen. Für unser Vorhaben hat sich das einleitend dargestellte Verständnis von direkter Verdrängung („direct displacement“; Marcuse 1985: 205) als ein durch Wohnungskündigung erzwungener Auszug als sinnvoll erwiesen. Wir lösen uns zudem von einem gewissermaßen personalisierten Verdrängungsverständnis, welches davon ausgeht, dass eine wohlhabendere Gruppe eine ärmere Mieter_innschaft verdrängt (siehe u. a. Alisch/Zum Felde 1990: 277; Helbrecht 2016: 9). Suggeriert wird damit, dass Gentrifizierung respektive Verdrängung als aktiver Akt von zahlungskräftigen Mieter_innen selbst vollzogen wird. Das trifft allerdings nicht für jede Form von Verdrängung im Kontext von Gentrifizierung zu.

Mit dieser Präzisierung geht ein spezifisches Verständnis von Gentrifizierung einher: Hier liegt das Augenmerk auf stadtteilbezogenen Aufwertungsprozessen und Strategien unterschiedlicher Akteur_innen (siehe einleitend) und dem damit einhergehenden „Austausch der Bevölkerung“ (Holm 2018: 152). Offen bleibt jedoch, wer diesen Austausch verantwortet beziehungsweise erzeugt. Das ist für Verdrängungsformen wichtig, bei denen nicht neue Mieter_innen die bisherigen verdrängen, sondern vielmehr die bisherige Mieter_innenschaft durch Praktiken der institutionellen Investoren, teils legitimiert durch politische Bestrebungen, verdrängt werden. Im Falle von ,Leerkündigungen“ und den darauffolgenden Aufwertungen und Mietsteigerungen für Neuverträge ziehen nämlich zahlungskräftigere Personen erst in einem zweiten Schritt in die sanierten oder neu gebauten Wohnungen ein. Die Haushalte, die erzwungenermaßen wegziehen mussten, könnten - selbst wenn sie erneut aus einer zwischengenutzten Wohnung umziehen würden - in der Regel die neuen Mieten nicht mehr bezahlen („exclusionary displacement“; Marcuse 1985: 205) beziehungsweise sind nicht dazu bereit. Hierfür sind jedoch die baulichen Aufwertungen ursächlich und nicht die neu zuziehenden Mieter_innen.

Eine weitere Präzisierung des Verständnisses von Verdrängung ist zentral: Im Projekt fokussierten wir nicht nur Personen, die bereits weggezogen sind (also bereits verdrängt wurden), sondern auch solche, denen der Wohnungsverlust noch bevorsteht und auf die ein Verdrängungsdruck (,displacement pressure“; ebd.) ausgeübt wird. 
Neben diesen konzeptionell-begrifflichen Präzisierungen liegt es vor dem beschriebenen Hintergrund nahe, von einem prozessualen Verdrängungsverständnis auszugehen, mit dem die zeitliche Spanne vor, während und nach dem Ausspruch der Kündigungen in den Blick genommen und der Wandelbarkeit über die Zeit hinweg Rechnung getragen werden kann (Sakizlioğlu 2014). Die empirischen Ergebnisse des Projekts WOHNSOG legen nahe, dass ein prozessuales Verständnis von Umgang, von dem in den methodologischen Prämissen der Grounded Theory im Sinne Strauss' und Corbins (1996) ausgegangen wird, gegenstandsangemessen ist.

Schließlich - und das ist durchaus auch als forschungspraktisches Argument zu verstehen - stellt Verdrängung im Zuge derart motivierter ,Leerkündigungen ' oftmals großer Liegenschaften kein unsichtbares oder vernachlässigbares Phänomen dar. Vielmehr ist der bevorstehende oder bereits erlebte Wohnungsverlust der Mieter_innen immer häufiger medial-öffentlich omnipräsent und Gegenstand widerständiger Praktiken sozialer Bewegungen.

\section{Qualitative Operationalisierung des Umgangs mit} Verdrängungserfahrung

Mit Blick auf den Umgang mit Verdrängungserfahrung respektive einer Wohnungskündigung stellt sich die Frage, wie der Begriff ,Umgang' theoretisch fundiert und qualitativ operationalisiert werden kann. Im Projekt WOHNSOG steht Umgang für die konkreten Handlungen, Strategien und Interaktionen der interviewten Mieter_innen bezüglich der Erfahrung der Kündigung und des damit einhergehenden erzwungenen Auszugs respektive des gesamten Verdrängungsprozesses. Im Sinne Webers (1984) fokussieren wir nicht nur auf das Tun, sondern auch auf das Dulden oder Unterlassen. Ein solcher Fokus auf und ein Interesse am Umgang lässt sich forschungspraktisch sehr gut mittels des handlungstheoretischen Kodierparadigmas, das für die Grounded Theory im Sinne von Strauss und Corbin (1996: 78) wesentlich ist, qualitativ operationalisieren und erkenntnistheoretisch begründen. Strauss (1993) geht dabei von einer interaktionalen Handlungstheorie aus. Dem Kodierparadigma liegt die erkenntnistheoretische Annahme zugrunde, dass Sachverhalte, Ereignisse und Handlungen kausal miteinander verwoben und untereinander bedingt sind (Breuer 2010: 85), und zwar in einem relationalen Verständnis (Strübing 2008: 28) und jeweils abhängig vom aus der Forschungsfrage resultierenden Fokus auf das Datenmaterial. Das Kodierparadigma kann insofern forschungspraktisch als Heuristik dienen und ist Ausdruck einer abduktiven Haltung (Meuth 2018: 82; Tiefel 2016, 2005).

Forschungspraktisch bedeutet die Arbeit mit einem Kodierparadigma, dass an das empirische Material systematisch $W$-Fragen gestellt werden: Um welches Phänomen geht es in der vorliegenden Sequenz? Die Benennung des zentralen Phänomens einer Passage ist herausfordernd und oft erst nach ausführlicher Interpretation und Bearbeitung des Interviewtextes möglich. Welche ursächlichen Bedingungen führen zum Phänomen? Welche Kontextdaten spezifizieren das Phänomen? Welcher Umgang mit dem Phänomen ist zu verzeichnen (Handlungen, Strategien, Interaktionen)? Welche intervenierenden Bedingungen beeinflussen, ermöglichen oder behindern 
diesen Umgang? Welche Konsequenzen ergeben sich aus dem je spezifischen Umgang mit den einzelnen Phänomenen?

\section{Kontextualisierung der Subjektperspektive}

Indem qualitative Studien auf das Erleben der Wohnungskündigung und den Umgang damit fokussieren, machen sie die subjektiven Perspektiven und Deutungsmuster auf Verdrängungsprozesse rekonstruierbar (Mikroebene). Im Projekt WOHNSOG haben wir über die einzelnen Interviews hinweg sowie im Vergleich zwischen den Positionierungen der Mieter_innen in den verschiedenen Fallstudien heterogene Umgangsmuster herausarbeiten können. Durch die systematische Forschungspraxis mit dem Kodierparadigma sind die subjektbezogenen rekonstruierten Perspektiven und Umgangsmuster Teile komplexer Bedingungsgefüge und damit immer kontextualisiert. Neben Kontext- und Einflussfaktoren auf individueller Ebene werden somit auch förderliche sowie hinderliche Faktoren des Umgangs berücksichtigt, etwa strukturelle Faktoren. Das sind unter anderem mietrechtliche Regelungen, angespannte Wohnungsmärkte, ausbleibende Hilfsangebote, vorherrschende politische, soziale und ökonomische Machtverhältnisse, die Bedingungen der jeweiligen Totalsanierungs- oder Ersatzneubauprojekte, die Praktiken der Verwaltungen und Eigentümerschaften, aber auch soziokulturelle Werte, Normen und Rollenbilder, die den symbolischen Orientierungsrahmen für den Umgang darstellen (Meso- und Makroebene).

Neben der forschungspraktischen Arbeit mit dem Kodierparadigma können derart rekonstruierte Perspektiven auf und Umgangsmuster mit Verdrängung schließlich mit dem methodischen Prinzip der Fallstudien weiter kontextualisiert werden (siehe Kapitel 3).

Die erkenntnistheoretischen Annahmen zum Bedingungsgefüge und zur Kontextualisierung der untersuchten Phänomene, wie sie in der Grounded Theory (Strauss/Corbin 1996) gegeben sind, erweisen sich insofern als gewinnbringend für eine kontextualisierte subjektive Perspektive auf Verdrängung, da die kontextuelle Verortung stets in den Analyseprozess eingewoben ist und nicht etwa nachgelagert und summativvorgenommen wird. Die Perspektiven und Umgangsmuster der Mieter_innen sind über die je spezifischen „biografischen Auseinandersetzungs- und Gestaltungsprozesse“ (Wiezorek 2016: 185) hinaus immer innerhalb ihres bedingenden Rahmens, der nicht nur auf der Mikroebene verortet ist, erfassbar. Es ist deshalb davon auszugehen, dass derart vorgenommene Analysen von Perspektiven und Umgangsmustern nicht nur das Ergebnis individueller Entscheidungen sind, sondern abhängig von einer ,jeweiligen raum-zeitlichen und sozialen Gebundenheit“" (Strübing 2008: 38) sind und damit kontextualisiert vorgenommen werden.

Wir gehen also davon aus, dass differenzierte und methodisch-methodologisch durchdachte qualitative Studien neben der Innensicht aus der „Froschperspektive“ (Eckardt 2018: 22) respektive mit dem Fokus auf lokale mikro-soziologische Prozesse (ebd.) stets auch Erkenntnisse struktureller Art zum Forschungsfeld liefern (Sakizlioğlu 2014: 17f.; Wießner 1990); seien das Einblicke in das ,Entmietungsmanagement ' der Eigentümerschaften, Mechanismen sozialer Ungleichheit und Machtverhältnisse oder auch 
politische Prozesse, um nur einige Beispiele zu benennen, die jenseits der persönlichen Interaktionsebene der Verdrängten auf der mikroanalytischen Ebene verortet sind. Entscheidend ist, dass die Blickrichtung auf weitere Aggregatsebenen (Meso- und Makroebene) durch derartige qualitative Studien jeweils vom Standpunkt der Subjekte (also der Verdrängten) aus vorgenommen werden.

Ein solches methodisch und methodologisch begründetes Forschungsdesign plausibilisiert, dass der Blick auf die Perspektiven und den Umgang mit der Wohnungskündigung respektive der Verdrängungserfahrung zwar vom Erleben, von den Meinungen und Wahrnehmungen der Mieter_innen aus vorgenommen wird - deshalb der Zusatz subjektorientiert. Deutlich sollte aber auch geworden sein, dass dies nicht mit einer subjektivistischen und individualisierenden Analyse von Verdrängungserfahrungen zu verwechseln ist. Letzteres birgt nämlich die Gefahr, dass es zu einer Individualisierung der Verdrängungsfrage kommt, und zwar in dem Stil, dass Forschungen Erkenntnisse liefern, wie der Umgang mit und die Bewältigung von Verdrängung positiv gestaltet oder optimiert werden könnte, zum Beispiel durch Selbstoptimierung und Arbeit am Selbst der Betroffenen. Eine Tendenz, die in der Stadtforschung aus wohnpolitischen und residualen Logiken heraus bekannt ist, die aber auch in einer individualisierenden Problembearbeitung Sozialer Arbeit zum Ausdruck kommt.

\section{Forschung als machtvoller Prozess und reflexive Positionalität}

Schließlich möchten wir die in der (Verdrängungs-)Forschung präsenten gesellschaftlichen Machtverhältnisse nicht unerwähnt lassen: Es wurde immer wieder deutlich, dass Verdrängungsforschung ein „gesellschaftlich vermittelter, das heißt ein in Herrschaftsverhältnisse eingebundener und in spezifische Interessens- und Konfliktkonstellationen verstrickter sozialer Prozess" (Anhorn/Stehr 2012: 57) ist und es sich zudem um ein normativ aufgeladenes Forschungsfeld handelt (Eckardt 2018: VII). Bereits die Definitionen sowie die Operationalisierungen von Verdrängung beziehungsweise Gentrifizierung bedingen den Zugang zum Gegenstand und haben politische Implikationen. „Denn Gentrification ist gewiss kein reibungsloser, konfliktloser Prozess, den man quasi einfach nur wissenschaftlich distanziert beobachten könnte“ (Helbrecht 2016: 11). Genau deshalb, aber auch aufgrund der in Kapitel 2 diskutierten blinden Flecken, die sich aus der Positionalität der Forscher_innen ergeben, erweist sich eine reflexive Positionalität, oder mit Allen (2008: 180) gesprochen die „referential reflexivity“, als äußerst wichtig.

Im Projekt erwies es sich als erkenntnisgenerierend, über folgende Positionalitäten zu reflektieren: Disziplinäre Positionalität und Präkonzepte, forschungspraktische und forschungsethische Aspekte fordern die professionelle Positionalität der Forscher_in, die(Ohn-)Mächtigkeit der Forscher_in im Untersuchungsfeld sowie die Reflexivität der Emotionen, die dabei hilft, die Positionalität der Forscher_in im Analyseprozess leichter explizieren zu können (Meuth 2018: 93 f.; siehe ausführlich Kapitel 9 in Meuth/ Reutlinger 2021). Beispielsweise expliziert die Reflexion über die disziplinären Perspektiven des Projektteams, dass ausgehend von bestimmten 
Präkonzepten und der wissenschaftlichen Sozialisation der untersuchte Gegenstand in einer spezifischen Weise fokussiert wird: Der Blick wird auf die Perspektiven der von Verdrängung Betroffenen gerichtet, die von ihnen erlebten sozialen Ungleichheiten werden dargelegt. Verdichtungspolitik und ,Leerkündigungen' hätten denn auch ganz anders thematisiert werden können, zum Beispiel als lukrative Finanzanlagen oder im Sinne von Fragen, wie man Entmietung aus einer finanziellen und dienstleistungsbezogenen Sichtweise optimieren könnte.

Die Reflexion der eigenen (Ohn-)Mächtigkeit der Forscher_in im Untersuchungsfeld förderte beispielsweise die eigenen Verstrickungen mit dem fokussierten Gegenstand sowie den erlebten Ambivalenzen zutage. Das Bewusstsein für ungleich verteilte Machtpositionen der beteiligten Akteur_innen forderte im Forschungsprozess immer wieder die wissenschaftliche wie auch die politische Positionalität der Forscher_innen heraus sowie einen bewussten Umgang mit der eigenen Involviertheit und (Ohn-) Mächtigkeit. Dazu ist die privilegierte und handlungsentlastende Position der Forscher_innen zu reflektieren, aus der heraus Wissen allzu leicht auch als Herrschaftswissen genutzt werden kann. Zudem legt diese Reflexion offen, dass wir als Privatpersonen (unbewusst) Teil der kritisierten Prozesse und Probleme sind (Eckardt 2018: 2). Ein solches Dilemma, das uns als Forschende in diesem Feld ohnmächtig zurücklässt, besteht darin, dass wir, als pensionskassenpflichtige Arbeitnehmer_innen,[7] wenn auch sehr subtil und indirekt, selbst in Bau- und Sanierungsprojekte institutioneller Eigentümerschaften und damit produzierte Verdrängungen verstrickt sind.

Nicht nur um dem machtvollen Forschungsprozess zu Verdrängung sowie den eigenen Positionalitäten Rechnung zu tragen, stellt sich eine reflexive Positionalität als wichtig heraus, sondern auch aufgrund einer epistemologischen Haltung, mit der anerkannt wird, dass sozialwissenschaftliche Forschung „nicht durch eine universale monolithische Methodologie“ gekennzeichnet ist, „sondern durch eine Vielfalt von Erkenntnisvarianten“ (Breuer 2010: 40). Insofern ist es unserer Ansicht nach wichtig, dem „selbstbezüglichen Charakter des Erkenntnisprozesses“(ebd.) methodisch sensibilisiert und methodologisch begründet Rechnung zu tragen.

\section{Fazit}

Ziel des vorliegenden Artikels ist es, vor dem Hintergrund des Forschungsprojektes WOHNSOG einen exemplarischen Beitrag zu konzeptionellen und methodisch-methodologischen Fragen und Herausforderungen sowie möglichen Lösungswegen für einen qualitativen Zugang zu Verdrängung zu leisten. Damit reagieren wir erstens auf die alltagspraktische Präsenz vielfältiger und immer zahlreicherer Verdrängungserfahrungen und das Plädoyer in der Gentrifizierungsforschung, Verdrängung verstärkt aufdie und aus der Perspektive der Betroffenen zu erforschen (Holm 2013: 59). Zweitens stellt sich die Fragestellung dieses Artikels mit Blick auf konzeptionelle und methodisch-methodologische Verunsicherungen in der Fachliteratur als zentral heraus.

Zunächst haben wir aufgezeigt, wie Verdichtungspolitiken in der Schweiz, Aufwertungsbestrebungen unter den aktuellen Bedingungen auf dem Mietmarkt und Praktiken der ,Leerkündigungen“ eine diskursive 
Gemengelage für eine spezifische Form direkter Verdrängung mittels massenhafter Wohnungskündigungen innerhalb einzelner Siedlungen darstellt.

In einem zweiten Schritt haben wir den Diskurs um Gentrifizierung hinsichtlich des Aspektes von Verdrängung in groben Zügen dargelegt. Deutlich wurde, dass die Ursache für eine relative Vernachlässigung von Verdrängung in diesem Diskurs mitunter auf konzeptionelle, aber auch auf methodische und methodologische Herausforderungen selbst zurückgeführt wird.

Drittens haben wir das Forschungsdesign des Projekts WOHNSOG mit dem Fokus auf die Perspektive der Mieter_innen auf ihre Wohnungskündigung als Form direkter Verdrängung im schweizerischen Kontext vorgestellt.

Darauf aufbauend haben wir in einem vierten Schritt unter Berücksichtigung der aufgerissenen Problemlagen sowohl des Diskurses als auch des im WOHNSOG untersuchten Forschungsgegenstandes konzeptionelle und methodisch-methodologische Schlussfolgerungen für qualitative Zugänge zu Verdrängung vorgenommen. Damit zeigten wir mittels exemplarischer Bezüge und Diskussionen aus dem Projektzusammenhang Lösungswege auf, die Denkanstöße für die Herausforderungen zukünftiger Projekte qualitativer Verdrängungsforschung bieten können.

Mit dem vorliegenden Artikel sollte ferner deutlich geworden sein, was qualitative Studiendesigns bezüglich Verdrängung zu erfassen in der Lage sind. Aber auch Herausforderungen und Grenzen wurden aufgezeigt. Wichtig erscheint uns, dass die unterschiedlichen erkenntnistheoretischen wie forschungspraktischen Zugänge - also qualitativ und quantitativ - nicht gegeneinander ausgespielt werden sollten, da das „konzeptionelle Spannungsverhältnis“ ernst zu nehmen ist und „nicht einseitig aufgelöst werden kann, sondern durch Problemzentrierung fruchtbar gemacht werden muss“ (Eckardt 2018: 22). Forschungspraktisch bedeutet dies aus unserer Sicht, dass die Methoden gegenstandsangemessen zu wählen sind, also abhängig vom Erkenntnisinteresse, dem fokussierten Gegenstand und der Fragestellung. Gerade wegen des normativen Gehalts des Themas Verdrängung ist es unserer Ansicht nach in qualitativen Studien gewinnbringend, stark narrative Ansätze aufzugreifen, um die Perspektive der Betroffenen nicht zu sehr zu strukturieren und zu steuern, sondern ihren eigenen Deutungen, Setzungen und Erfahrungen mehr Raum zu lassen.

Vor dem Hintergrund des einschlägigen wissenschaftlichen Diskurses zu Verdrängung im Kontext von Gentrifizierung sowie basierend auf den Erfahrungen des Projekts WOHNSOG gehen wir von der Notwendigkeit einer kritischen Verdrängungsforschung aus, die sowohl Verdrängungsprozesse strukturell erforscht als auch dezidiert die subjektorientierte Perspektive der Verdrängten in den Fokus rückt. Das erscheint uns mit Blick auf die in der kritischen Stadtforschung formulierten These der (stadtpolitischen) Diskursverschiebung im Feld der Gentrifizierung (Slater 2006; Holm 2013; Newmann/Wyly 2006; Shaw 2008; Shaw/Hagemans 2015; Smith 2008) als zentral. Wird der Aspekt der Verdrängung als Teil von Gentrifizierungsprozessen in der Stadt- und Raumplanung, aber auch im Fachdiskurs beschönigt oder gar negiert, stellt das für Holm (2013: 54) einen „Verharmlosungsdiskurs“ dar. Vorstellungen, nach denen es durch bauliche und soziale Aufwertungen zur sozialen Mischung käme, von der auch ärmere Menschen profitierten, bezeichnet er als „Romantisierungsdiskurs“ (ebd.). Nimmt 
man diese These ernst, so ergibt sich die dringende Notwendigkeit einer differenzierten Debatte um Konzepte, Methoden und Methodologien der Verdrängungsforschung. Speziell empirische, methodisch-methodologische und theoretische Auseinandersetzungen mit den Perspektiven von Menschen mit Verdrängungserfahrung könnten unseres Erachtens einen Beitrag dazu leisten, solcherlei Diskursen nicht weiter Vorschub zu leisten.

Dieser Artikel wurde durch Publikationsmittel des Schweizerischen Nationalfonds zur Förderung der wissenschaftlichen Forschung (SNF) gefördert.

\section{Endnoten}

[1] Ein herzliches Dankeschön gilt der Redaktion von s u b $\backslash \mathrm{u}$ r b a n sowie den anonymen Gutachter_innen für die herausfordernden, aber konstruktiven Rückmeldungen zu Vorversionen dieses Artikels.

[2] Für Ausführungen zu Verdrängung unabhängig von Gentrifizierung (,displacement sans gentrification“) siehe Pull et al. (2021: 7 f.).

[3] Zur Perspektive von Betroffenen auf Verdrängung siehe für neuere Studien u. a. Atkinson (2015); Bernien (2017); Debrunner (o. J.); Kasiske (2014); Sakizlioğlu (2014); Üblacker/ Lukas (2019); Valli (2015). Verdrängung durch den Abriss sozialer Wohnungsbauten untersuchen für die USA und die Niederlande Kleinhans/van der Laan Bouma-Doff (2008); Kleinhans (2003). Zum Thema Verdrängung im Zuge von Zwangsräumungen siehe u. a. Berner/Holm/Jensen (2015); Türkmen (2015).

[4] Das interdisziplinäre Projektteam setzte sich wie folgt zusammen: Co-Projektleitung: Christian Reutlinger (Erziehungswissenschaft, Humangeografie) und Miriam Meuth (Erziehungswissenschaft); Mitarbeit: Heidi Furrer (Soziale Arbeit), Patricia Roth (Urban Design), Nicola Hilti (Soziologie), Eva Lingg (Architektur); Projektlaufzeit: April 2017 bis Oktober 2020 (mit einjähriger Unterbrechung).

[5] Die Forschungsfragestellung wurde im Projektverlauf sukzessive fokussiert und präzisiert, was auch die leichten Varianzen in vorherigen Publikationen des Projekts erklärt.

[6] Eine geringe Leerstandsquote - also ein geringer Anteil leerstehender Wohnungen am Gesamtwohnungsbestand - stellt Beran und Nuissl (2019a: 15 f.) zufolge einen Hinweis auf angespannte Wohnungsmärkte dar.

[7] In der Schweiz besteht für Arbeitnehmer_innen eine Pensionskassenpflicht: Für alle volljährigen Angestellten, deren jährliches Einkommen mehr als 21.330 Schweizer Franken beträgt (gültig für das Jahr 2019), existiert eine obligatorische Altersvorsorge. Damit sind rund 80 Prozent der steuerpflichtigen Personen (BFS 2015) indirekt in die Praktiken der Pensionskassen involviert.

\section{Autor_innen}

Meuth Miriam ist Erziehungswissenschaftlerin mit Fokus auf Kinder- und Jugendhilfe/ Übergangsforschung, Wohnforschung und qualitative Sozialforschung. miriam.meuth@uzh.ch

Christian Reutlinger ist Sozialgeograph und Erziehungswissenschaftler mit Fokus auf Wohnen und Nachbarschaften, Öffentliche Räume und Gemeinwesen.

christian.reutlinger@ost.ch 


\section{Literatur}

Alisch, Monika / Zum Felde, Wolfgang (1990): „Das gute Wohngefühl ist weg!“. Wahrnehmungen, Bewertungen und Reaktionen von Bewohnern im Vorfeld von Verdrängungen. In: Jörg Blasius / Jens S. Dangschat (Hg.), Gentrification. Die Aufwertung innenstadtnaher Wohnviertel. Frankfurt am Main / New York: Campus Verlag, 277-324.

Allen, Chris (2008): Gentrification 'research' and the academic nobility. A different class? In: International Journal of Urban and Regional Research 32, 180-185.

Anhorn, Roland / Stehr, Johannes (2012): Grundmodelle von Gesellschaft und soziale Ausschliessung. Zum Gegenstand einer kritischen Forschungsperspektive Sozialer Arbeit. In: Elke Schimpf / Johannes Stehr (Hg.), Kritisches Forschen in der Sozialen Arbeit. Gegenstandsbereiche, Kontextbedingungen, Positionierungen, Perspektiven. Wiesbaden: Springer VS, 57-76.

Atkinson, Rowland (2000): Measuring gentrification and displacement in Greater London. In: Urban Studies 37/1, 149-165.

Atkinson, Rowland (2015): Losing one's place. Narratives of neighbourhood change, market injustice and symbolic displacement. In: Housing, Theory and Society 32/4, 373-388.

Beran, Fabian / Nuissl, Henning (2019a): Der Ausgangspunkt der Verdrängungsforschung. Angespannte Wohnungsmärkte. In: Wüstenrot Stiftung (Hg.), Verdrängung auf angespannten Wohnungsmärkten. Das Beispiel Berlin. Ludwigsburg: Wüstenrot Stiftung, 14-17.

Beran, Fabian / Nuissl, Henning (2019b): Verdrängung als Thema der Stadtforschung. In: Wüstenrot Stiftung (Hg.), Verdrängung auf angespannten Wohnungsmärkten. Das Beispiel Berlin. Ludwigsburg: Wüstenrot Stiftung, 18-21.

Berner, Laura / Holm, Andrej / Jensen, Inga (2015): Zwangsräumungen und die Krise des Hilfesystems. Eine Fallstudie in Berlin. Berlin.

Bernien, Sandra (2017): Verdrängung hat viele Gesichter. Über städtische Verdrängungsprozesse am Beispiel des Berliner Kaskelkiezes. In: Uwe Altrock / Ronald Kunze (Hg.), Stadterneuerung und Armut. Wiesbaden: Springer Fachmedien, 37-63.

Betancourt, Camilo (2016): Wieso - Weshalb - Wohin? Wohnbiographien als Instrument der sozialräumlichen Verdrängungsanalyse. In: Ilse Helbrecht (Hg.), Gentrifizierung in Berlin. Verdrängungsprozesse und Bleibestrategien. Bielefeld: transcript, 257-286.

Brake, Klaus / Herfert, Günter (Hg.) (2012): Reurbanisierung. Materialität und Diskurs in Deutschland. Wiesbaden: Springer VS.

Breuer, Franz (2010): Reflexive Grounded Theory. Eine Einführung für die Forschungspraxis. Wiesbaden: VS Verlag für Sozialwissenschaften.

Bundesamt für Raumentwicklung Schweiz: Bundesgesetz über die Raumplanung. Raumplanungsgesetz RPG.

Debrunner, Gabriela (o. J.): ResiDENSE - Lokale Governance der Verdichtung zur Steuerung von sozialen Exklusionsprozessen im Schweizer Mietwohnungsmarkt. Bern.

Debrunner, Gabriela / Hartmann, Thomas (2020): Strategic use of land policy instruments for affordable housing. Coping with social challenges under scarce land conditions in Swiss cities. In: Land Use Policy 99, 1-12.

Debrunner, Gabriela / Hengstermann, Andreas / Gerber, Jean-David (2020): The business of densification. Distribution of power, wealth and inequality in Swiss Policy Making. In: Town Planning Review 91/3, 259-281.

Easton, Sue / Lees, Loretta / Hubbard, Phil / Tate, Nicholas (2019): Measuring and mapping displacement. The problem of quantification in the battle against gentrification. In: Urban Studies 61/2, 1-21.

Eckardt, Frank (2018): Gentrifizierung. Forschung und Politik zu städtischen Verdrängungsprozessen. Wiesbaden: Springer VS.

Ertelt, Greta / Schulz, Carlotta-Elena / Thieme, Georg / Uhlig, Christiane (2016): Die statemade-rental-gap. Gentrification im Sozialwohnungsbau. In: Ilse Helbrecht (Hg.), Gentrifizierung in Berlin. Verdrängungsprozesse und Bleibestrategien. Bielefeld: transcript, 107-150.

Friedrichs, Jürgen (1996): Gentrification. Forschungsstand und methodologische Probleme. In: Jürgen Friedrichs / Robert Kecskes (Hg.), Gentrification. Theorie und Forschungsergebnisse. Opladen: Leske + Budrich, 13-40. 
Furrer, Heidi / Hilti, Nicola / Lingg, Eva / Meuth, Miriam / Roth, Patricia (2020): Solidarisierung und Entsolidarisierung von Mieterinnen und Mietern im Kontext von bedrohtem Wohnen. Zum Umgang mit ,Entmietungsstrategien‘. In: Stefan Paulus / Christian Reutlinger / Eleni Rutz-Spiroudis / Steve Stiehler / Sibille Hartmann / Sabine Makowka (Hg.), Mechanismen der Sozialen Frage. Hin- und Ableitungen zur Sozialen Arbeit. Berlin: Frank \& Timme, 231-242.

Grier, George / Grier, Eunice (1980): Urban displacement. A reconaissance. In: Shirley Bradway Laska / Daphne Spain (Hg.), Back to the city. Issues in neighborhood renovation. New York: Pergamon Press, 252-268.

Heeg, Susanne (2013): Wohnungen als Finanzanlage. Auswirkungen von Responsibilisierung und Finanzialisierung im Bereich des Wohnens. In: sub \urban. zeitschrift für kritische stadtforschung 1/1, 75-99.

Helbrecht, Ilse (2016): Gentrification und Verdrängung. In: Ilse Helbrecht (Hg.), Gentrifizierung in Berlin. Verdrängungsprozesse und Bleibestrategien. Bielefeld: transcript, 9-16.

Helfferich, Cornelia (2011): Die Qualität qualitativer Daten. Manual für die Durchführung qualitativer Interviews. Wiesbaden: VS Verlag für Sozialwissenschaften.

Hochschule Luzern (2019): Bauliche Verdichtung und Nutzungsverdichtung. Eine explorative und fallstudienbasierte Analyse. https://www.bwo.admin.ch/bwo/de/home/Wohnungsmarkt/studien-und-publikationen/bauliche-verdichtung.html (letzter Zugriff am 3.7.2020).

Holm, Andrej (2011): Wohnung als Ware. Zur Ökonomie und Politik der Wohnungsversorgung. In: Widersprüche 31/121, 9-20.

Holm, Andrej (2013): Wir bleiben alle! Gentrifizierung. Städtische Konflikte um Aufwertung und Verdrängung. Münster: Unrast.

Holm, Andrej (2018): Gentrification. In: Bernd Belina / Matthias Naumann / Anke Strüver (Hg.), Handbuch Kritische Stadtgeographie. Münster: Westfälisches Dampfboot, 152-157.

Kasiske, Florian (2014): „Aus dem Leben rausgerissen“. Verdrängung im Kontext von Gentrifizierung in St. Pauli-Süd. Hamburg.

Kleinhans, Reinout (2003): Displaced but still moving upwards in the housing career? Implications of forced residential relocation in the Netherlands. In: Housing Studies 18/4, 473-499.

Kleinhans, Reinout / van der Laan Bouma-Doff, Wenda (2008): On priority and progress. Forced residential relocation and housing chances in Haaglanden, the Netherlands. In: Housing Studies 23/4, 565-587.

Knabe, Judith / Leitner, Sigrid (2017): Soziale Arbeit, Sozial- und Wohnungspolitik. Ein unübersichtliches Feld. Ausschließungen vom Wohnungsmarkt und ihre Bewältigung. In: Sozialer Fortschritt 66/3-4, 229-247.

Langer, Antje (2010): Transkribieren. Grundlagen und Regeln. In: Barbara Friebertshäuser / Antje Langer / Annedore Prengel (Hg.), Handbuch qualitative Forschungsmethoden in der Erziehungswissenschaft. Weinheim und München: Beltz Juventa, 515-526.

Lees, Loretta / Slater, Tom / Wyly, Elvin K. (Hg.) (2010): The gentrification reader. London: Routledge.

Marcuse, Peter (1985): Gentrification, abandonment, and displacement. Connections, causes, and policy responses in New York City. In: Journal of Urban and Contemporary Law 28/1, 195-240.

Meuser, Michael / Nagel, Ulrike (2009): Experteninterviews und der Wandel der Wissensproduktion. In: Alexander Bogner / Beate Littig / Wolfgang Menz (Hg.), Experteninterviews. Theorien, Methoden, Anwendungsfelder. Wiesbaden: VS Verl. für Sozialwissenschaften, 35 .

Meuth, Miriam (2018): Wohnen. Erziehungswissenschaftliche Erkundungen. Weinheim/ Basel: Beltz Juventa.

Meuth, Miriam / Reutlinger, Christian (2020): (Drohenden) Wohnungsverlust bewältigen. Zum Umgang mit Gentrifizierungs- und Verdrängungserfahrungen. In: Gerd Stecklina / Jan Wienforth (Hg.), Handbuch: Lebensbewältigung und Soziale Arbeit. Praxis, Theorie und Empirie. Weinheim/Basel: Beltz Juventa, 614-622.

Meuth, Miriam / Reutlinger, Christian (2021): ,Entmietet‘ und verdrängt werden. Bielefeld: transcript.

Mieterinnen- und Mieterverband Ostschweiz (2018): Umbauten, Renovationen, Totalsanierungen. Eine Informationsbroschüre des Mieterinnen- und Mieterverbandes Ostschweiz. St. Gallen: Mieterinnen- und Mieterverband Ostschweiz. 
Newmann, Kathe / Wyly, Elvin K. (2006): The right to stay put, revisited. Gentrification and resistance to displacement in New York City. In: Urban Studies 43/1, 23-57.

Nuissl, Henning / Beran, Fabian (2019): Einleitung. In: Wüstenrot Stiftung (Hg.), Verdrängung auf angespannten Wohnungsmärkten. Das Beispiel Berlin. Ludwigsburg: Wüstenrot Stiftung, 8-11.

Przyborski, Aglaja / Wohlrab-Sahr, Monika (2009): Qualitative Sozialforschung. Ein Arbeitsbuch. München: Oldenbourg.

Pull, Emil / Baeten, Guy / Listerborn, Carina / Persdotter, Maria (2021): Introduction. Housing displacement. Conceptual and methodological Issues. In: Guy Baeten / Carina Listerborn / Maria Persdotter / Emil Pull (Hg.), Housing displacement. London / New York: Routledge, 1-17.

Reutlinger, Christian(2015): Innenentwicklung von Ortskernen und demokratische Prozesse. Einleitende Betrachtungen. In: Christian Reutlinger / Caroline Fritsche / Markus Markstaler / Andrea Schemmel / Martin Schlatter / Frieder Voll (Hg.), Vom Zwischeneinander der Disziplinen. Neue Perspektiven auf Siedlungs-Verdichtung. St.Gallen: FHO, 51-71.

Reutlinger, Christian / Furrer, Heidi / Hilti, Nicola / Lingg, Eva / Meuth, Miriam / Roth, Patricia (2019): Perspektive: Wohnungsverlust. Untersuchung zur sozialen Seite baulichplanerischer Strategien. In: Soziale Passagen 11/1, 215-219.

Sakizlioğlu, Bahar (2014): A comparative look at residents displacement experiences. The case of Amsterdam and Istanbul. Utrecht.

Schröer, Sebastian / Schulze, Heike (2010): Grounded Theory. In: Karin Bock / Ingrid Miethe (Hg.), Handbuch qualitative Methoden in der sozialen Arbeit. Opladen: Barbara Budrich, 277-288.

Schütze, Fritz (1983): Biographieforschung und narratives Interview. In: Neue Praxis 13/3, 283-293.

Schütze, Fritz (1984): Kognitive Figuren des autobiographischen Stegreiferzählens. In: Martin Kohli / Günther Robert (Hg.), Biographie und soziale Wirklichkeit. Neue Beiträge und Forschungsperspektiven. Stuttgart: Metzler, 78-117.

Shaw, Kate S. (2008): A response to 'The eviction of critical perspectives from gentrification research'. In: International Journal of Urban and Regional Research 32/1, 192-194.

Shaw, Kate S. / Hagemans, Iris W. (2015): 'Gentrification without displacement' and the consequent loss of place. The effects of class transition on low-income residents of secure housing in gentrifying areas. In: International Journal of Urban and Regional Research $39 / 2,323-341$.

Slater, Tom (2006): The eviction of critical perspectives from gentrification research. In: International Journal of Urban and Regional Research 30/4, 737-757.

Smith, Neil (2008): On 'The eviction of critical perspectives'. In: International Journal of Urban and Regional Research 32/1, 195-197.

Strauss, Anselm L. (1993): Continual permutations of action. New York: De Gruyter.

Strauss, Anselm L. / Corbin, Juliet (1996): Grounded Theory. Grundlagen qualitativer Sozialforschung. Weinheim: Beltz Psychologie-Verl.-Union.

Strübing, Jörg (2008): Grounded Theory. Zur sozialtheoretischen und epistemologischen Fundierung des Verfahrens der empirisch begründeten Theoriebildung. Wiesbaden: Springer VS.

Strübing, Jörg (2010): Grounded Theory. Ein pragmatischer Forschungsstil für die Sozialwissenschaften. In: Sabine Maschke / Ludwig Stecher (Hg.), Enzyklopädie Erziehungswissenschaft Online - EEO. Fachgebiet: Methoden der empirischen erziehungswissenschaftlichen Forschung, Wissenschaftstheoretische Grundlagen, Methodologie. Weinheim/ München: Juventa, 1-38.

Tiefel, Sandra (2005): Kodierung nach der Grounded Theory lern- und bildungstheoretisch modifiziert. Kodierungsleitlinien für die Analyse biographischen Lernens. In: ZBBS 6/1, 65-84.

Tiefel, Sandra (2016): Lebensbewältigung als Heuristik in qualitativen Forschungsdesigns. In: John Litau / Andreas Walther / Annegret Warth / Sophia Wey (Hg.), Theorie und Forschung zur Lebensbewältigung. Methodologische Vergewisserungen und empirische Befunde. Weinheim/Basel: Beltz Juventa, 88-107.

Türkmen, Ceren (2015): Neoliberaler Wohnungsmarkt zwischen sozialer Transformation und politischer Bewegung. In: sub \urban. zeitschrift für kritische stadtforschung 3/3, $125-136$. 
Üblacker, Jan / Lukas, Tim (2019): Keine Angst, es ist nur Gentrification? Soziale und ökonomische Ängste, Kriminalitätsfurcht und Verdrängungsdruck im Düsseldorfer Bahnhofsviertel. In: sub\urban. zeitschrift für kritische stadtforschung 7/1-2, 93-114.

Valli, Chiara (2015): A sense of displacement:. Long-time residents' feelings of displacement in gentrifying Bushwick, New York. In: International Journal of Urban and Regional Research 39/6, 1191-1208.

Vollmer, Lisa (2019): Der Gentrifizierungsbegriff in wohnungspolitischen Protesten. Kommentar zu Neil Smiths „Für eine Theorie der Gentrifizierung: ,Zurück in die Stadt” als Bewegung des Kapitals, nicht der Menschen“ (2019 [1979]). In: sub\urban. zeitschrift für kritische stadtforschung 7/3, 113-118.

Weber, Max (1984): Soziologische Grundbegriffe. Tübingen: Mohr.

Wießner, Reinhard (1990): Soziale und strukturelle Folgen von Modernisierungen in innenstadtnahen Gebieten. In: Jörg Blasius / Jens S. Dangschat (Hg.), Gentrification. Die Aufwertung innenstadtnaher Wohnviertel. Frankfurt am Main/New York: Campus Verlag, 301-324.

Wiezorek, Christine (2016): Bewältigung. Bildung. Sozialisation? Zur sozialpädagogischen Perspektivierung von Praktiken und Prozessen der Lebensgestaltung. Ein Kommentar. In: John Litau / Andreas Walther / Annegret Warth / Sophia Wey (Hg.), Theorie und Forschung zur Lebensbewältigung. Methodologische Vergewisserungen und empirische Befunde. Weinheim/Basel: Beltz Juventa, 181-202.

Wüstenrot Stiftung (Hg.) (2019): Verdrängung auf angespannten Wohnungsmärkten. Das Beispiel Berlin. Ludwigsburg: Wüstenrot Stiftung.

\section{Affected by Gentrification. An Exemplary Contribution to the Discussion of Conceptual and Methodical-Methodological Questions of Qualitative Displacement Research}

Displacement as part of gentrification processes represents an important aspect of critical urban and housing studies that needs to be researched more intensively. A qualitative research approach provides an appropriate way to study the experiences and subjective interpretations of loss of home and displacement. In addition to conceptual and methodical-methodological questions and discussions, this phenomenon must also be examined empirically in a differentiated way, not least by taking into account how people threatened or affected by displacement look at, experience and deal with it. In this light, this article examines the question of which conceptual and methodical-methodological challenges and which possible solutions arise for a qualitative approach to displacement. Discussing this question based on a recently completed research project, we aim on contributing to the discussion of methods and ways of understanding qualitative displacement research in the context of gentrification. 